\title{
Tropical Cyclone Energetics and Structure
}

\author{
Kerry Emanuel \\ Program in Atmospheres, Oceans, and Climate \\ Massachusetts Institute of Technology
}

\section{Introduction}

Aircraft measurements of tropical cyclones that commenced during World War II allowed scientists of that era to paint the first reasonably detailed picture of the wind and thermal structure of tropical cyclones. This led to the first attempts to quantify the energy cycle of these storms and to understand the physical control of their structure. In this contribution, I review the history of research on the energy cycle and structure of tropical cyclones and offer a revised interpretation of their structure.

\section{Energetics}

The first reasonably accurate description of the energy cycle of tropical cyclones appeared in a paper by Herbert Riehl (1950). To the best of this author's knowledge, this is the first paper in which it is explicitly recognized that the energy source of hurricanes arises from the in situ evaporation of ocean water ${ }^{1}$. By the next year, another German scientist, Ernst Kleinschmidt, could take it for granted that "the heat removed from the sea by the storm is the basic energy source of the typhoon" (Kleinschmidt, 1951). Kleinschmidt also showed that thermal wind balance in a hurricane-like vortex, coupled with assumed moist adiabatic lapse rates on angular momentum surfaces, implies a particular shape of such surfaces. He assumed that a specified fraction, $q$, of the azimuthal velocity that would obtain if angular momentum were conserved in the inflow, is left by the time the air reaches the eyewall, and derived an expression for the maximum wind speed:

$$
v_{\max }^{2}=2 E \frac{q^{2}}{1-q^{2}}
$$

where $E$ is the potential energy found from a tephigram, assuming that air ascending in the eyewall has acquired some additional enthalpy from the ocean. Kleinschmidt did not provide a specific method for estimating this enthalpy increase, and (1) is sensitive to the arbitrary value of $q$ specified.

In his widely circulated textbook, now regarded as a classic, Riehl (1954) described hurricanes as heat engines and showed that for air ascending in the eyewall to be appreciably warmer than that of the distant environment, a condition for conversion of potential to kinetic energy, the inflowing air had to acquire enthalpy from the underlying surface.

\footnotetext{
${ }^{1}$ Byers (1944) recognized that the observation of nearly constant temperature following air flowing down the pressure gradient near the surface implies a sensible heat source from the ocean. The existence of isothermal inflow has been called into question by more recent observations.
} 
The work of Riehl and his colleagues, most notably Joanne Malkus, culminated in the publication of two papers in the early 1960's: Malkus and Riehl (1960) and Riehl (1963). The first of these once again emphasized that the horizontal temperature gradients that sustain tropical cyclones arise from heat transfer from the ocean. Making use of the observation that the horizontal pressure gradient is very weak at the top of the storm, that temperature lapse rates are very nearly moist adiabatic in the eyewall, and that the temperature of lifted parcels is a function of their boundary layer equivalent potential temperature, Malkus and Riehl (1960) used the hydrostatic relation to calculate a relationship between the surface pressure fall from the environment to the inner edge of the eyewall:

$$
\delta p_{s}=-2.5 \delta \theta_{e b}
$$

where $\delta p_{s}$ is the surface pressure drop in millibars, and $\delta \theta_{e b}$ is the increase in equivalent potential temperature, in Kelvins. In deriving this, the horizontal isobaric height gradient was assumed to vanish at $100 \mathrm{mb}$. This is a simple quantitative relationship showing explicitly the relationship between a measure of hurricane intensity and the increase in boundary layer entropy necessarily arising from sea-air enthalpy transfer. Riehl (1963) showed that (2) is well verified in observations of actual storms (with a best-fit coefficient of 2.56) and extended the Malkus and Riehl work in several ways. First, he made use of an argument made by Riehl and Malkus (1961) that outside the eyewall, where latent heat release is weak, conservation of potential vorticity integrated over a volume capped by an isentropic surface above the boundary layer leads to the conclusion that the curl of the surface stress must vanish, which for an axisymmetric vortex gives

$$
r \tau_{z \theta}=\text { constant }
$$

where $\tau_{z \theta}$ is the azimuthal component of the surface stress. (We will test this proposition in section 3.) Given that the stress varies nearly as the square of the wind speed, (3) implies that

$$
v_{\theta} \sim r^{-1 / 2}
$$

where $v_{\theta}$ is the azimuthal wind speed. Using (4) and assuming cyclostrophic balance gives an approximate expression for the pressure drop from some outer radius, $r_{o}$, to the radius of maximum azimuthal winds, $r_{i}$ :

$$
v_{\max }^{2} \simeq-\rho \delta p_{s}
$$

where $v_{\max }$ is the maximum azimuthal wind speed and $\rho$ is a mean air density in the boundary layer. Eliminating $\delta p_{s}$ between (5) and (2), and using an estimate of $\rho$ gives 


$$
v_{\max } \simeq 14.1\left(\delta \theta_{e b}\right)^{1 / 2}
$$

where $v_{\max }$ is in $m s^{-1}$.

In the next step, Riehl estimated $\delta \theta_{e b}$ from conservation of entropy and angular momentum in the inflow. I will slightly abbreviate and generalize his derivation here.

Assuming that both entropy $\left(\theta_{e}\right)$ and angular momentum $(M)$ are vertically uniform in the boundary layer, integration of the conservation equations for entropy and angular momentum through the depth of the boundary layer gives

$$
\psi \frac{\partial \theta_{e}}{\partial r}=C_{k}\left(\theta_{e s}-\theta_{e}\right) r|V|
$$

and

$$
\psi \frac{\partial M}{\partial r}=-r^{2} \tau_{z \theta}
$$

where $\psi$ is the mass streamfunction of the flow in the $r-z$ plane evaluated at the top of the boundary layer, $C_{k}$ is an enthalpy transfer coefficient, $\theta_{e s}$ is the saturation equivalent potential temperature of the sea surface, $|V|$ is a surface wind speed, and $M$ is the absolute angular momentum per unit mass, given by

$$
M=r v_{\theta}+\frac{1}{2} f r^{2}
$$

Eliminating $\psi$ between (7) and (8) gives

$$
\frac{\partial \theta_{e}}{\partial r}=-\frac{C_{k}\left(\theta_{e s}-\theta_{e}\right)}{r \tau_{z \theta}}|V| \frac{\partial M}{\partial r}
$$

Recall from (3) that $r \tau_{z \theta}$ is assumed constant. Also assuming that $|V| \simeq v_{\theta}$, we use (4) to express $v_{\theta}$ as

$$
v_{\theta}=v_{\max }\left(r_{m} / r\right)^{1 / 2}
$$

where $r_{m}$ is the radius of maximum winds. Using (9) for M, we can integrate (10) from $r_{m}$ to some outer radius, $r_{h}$, to get

$$
\delta \theta_{e b} \simeq \frac{C_{k}\left(\theta_{e s}-\theta_{e}\right)}{2 r \tau_{z \theta}} v_{\max }\left[v_{\max } r_{m} \ln \frac{r_{h}}{r_{m}}+\frac{4}{3} f r_{m}^{1 / 2}\left(r_{h}^{3 / 2}-r_{m}^{3 / 2}\right)\right]
$$


where we have assumed that $\left(\theta_{e s}-\theta_{e}\right)$ does not vary with radius. Now using $r \tau_{z \theta} \simeq r_{m} C_{D} v_{\max }^{2}$, where $C_{D}$ is the drag coefficient, we can write (11) as

$$
\delta \theta_{e b} \simeq \frac{C_{k}\left(\theta_{e s}-\theta_{e}\right)}{2 C_{D}}\left[\ln \frac{r_{h}}{r_{m}}+\frac{4}{3} \frac{f r_{m}}{v_{\max }}\left(\left(\frac{r_{h}}{r_{m}}\right)^{3 / 2}-1\right)\right] .
$$

Noting that, from (3) $r_{m} v_{\max }^{2}=r_{h} v_{h}^{2}$, where $v_{h}$ is the wind speed at radius $r_{h}$, making the approximation that $r_{m}<<r_{h}$, and substituting (12) into (6) gives

$$
v_{\max }^{2} \simeq 100 \frac{C_{k}}{C_{D}}\left(\theta_{e s}-\theta_{e}\right)\left[\ln \left(\frac{r_{h}}{r_{m}}\right)+\frac{4}{3} \frac{f r_{h}}{v_{h}}\right],
$$

which is equation (27) from Riehl (1963), except that Riehl assumed that $C_{D}=C_{k}$.

Note that this, together with $r_{m} v_{\max }^{2}=r_{h} v_{h}^{2}$ (from (3)), gives a transcendental equation for the maximum wind speed as a function of the degree of thermodynamic disequilibrium between the ocean and atmosphere, the Coriolis parameter, and the wind velocity at some specified radius. (Riehl goes on to make what in my view is a somewhat circular argument that there is another dynamical limit on the relationship between $v_{\max }$ and $r_{m}$ which, together with (13), determines the radius of maximum wind and an outer radius at the same time.) We shall show later that Riehl comes very close, in (13), to an energetic limit on hurricane intensity ${ }^{2}$.

${ }^{2}$ Malkus and Riehl (1960) came even closer. Their equation (33) invokes conservation of $\theta_{e}$ along a boundary layer streamline, yielding

$v \frac{\partial \theta_{e}}{\partial s}=C_{k} v \frac{\theta_{e s}^{*}-\theta_{e a}}{h}$,

where $\theta_{e s}^{*}$ is the saturation $\theta_{e}$ of the sea surface, $\theta_{e a}$ is the $\theta_{e}$ of the ambient boundary layer air, $h$ is the boundary layer depth, and the differentiation is along a streamline. Note that I have changed the notation for consistency, and that Malkus and Riehl unintentionally omitted the factor $h$. Combining this with (2) gives

$-v \frac{\partial p}{\partial s}=2.5 C_{k} v \frac{\theta_{e s}^{*}-\theta_{e a}}{h}$.

This is essentially the unnumbered equation after (33) in Malkus and Riehl. They also wrote down an expression (their equation 35) for conservation of energy along a streamline in the boundary layer:

$-v \frac{\partial p}{\partial s}=C_{D} \rho \frac{v^{3}}{h}$

Had they eliminated pressure between (a) and (b), they would have obtained the correct expression for maximum wind speed, our (21), albeit with fixed thermodynamic efficiency. Instead, they combined (b) with a balance equation for sensible heat along a boundary layer streamline, to obtain a peculiar relationship between maximum wind and air-sea temperature difference (their equation 36 ). 
Several years earlier, Miller (1958) developed a theory for the minimum central pressure in hurricanes. Miller also starts by assuming a moist adiabatic eyewall, but explicitly ignored any increase in entropy from the outer region into the eyewall, opting instead to assume that the eyewall air starts out at sea surface temperature and with a relative humidity of $85 \%$. Miller then estimated a vertical profile of temperature in the eye itself by assuming dry adiabatic descent modified by mixing with the eyewall air, along the line of reasoning explored by Malkus (1958). Once the eye temperature profile is constructed, the central surface pressure is calculated hydrostatically, assuming a level of zero horizontal pressure gradient at the standard pressure level nearest the level of neutral buoyancy for undilute pseudo-adiabatic ascent in the environment. The calculated central pressures were in good agreement with the minimum pressures recorded in a limited sample of intense hurricanes.

It is important to note here that Miller's work departs in a significant way from the line of reasoning adopted by Riehl and Malkus. The latter had emphasized the crucial importance of enthalpy transfer from the ocean, while Miller regarded the hurricane as resulting from the release of conditional instability of the ambient atmosphere, requiring no enhanced air-sea enthalpy flux. He quotes Byers (1944) statement that compared the hurricane to "one huge parcel of ascending air" and states in his opening sentence that "the principal source of energy of the tropical storm is the release of the latent heat of condensation", a statement rather precisely analogous to a claim that elevators are driven upward by the downward force on the counterweights: both statements are true but miss the point. In hindsight, Miller's estimate of the maximum intensity of hurricanes is energetically inconsistent. As the eyewall entropy is no larger than that of its environment, there can be no conversion of potential to kinetic energy by the overturning circulation of the storm; at the same time, the eye itself contains descending air with high temperature, a process that converts kinetic to potential energy. Thus Miller's energy cycle in the net absorbs rather than produces kinetic energy and thus cannot maintain the system against dissipation. In an important sense, Miller's analysis presaged the CISK thinking that became the dominant paradigm for tropical cyclone physics after the publication of Charney and Eliassen (1964). This thinking emphasizes the interaction between cumulus convection and the cyclone circulation rather than enthalpy flow from the ocean.

Meanwhile, the development of new observational tools and techniques continued apace. By the late 1960s, the axisymmetric structure of mature hurricanes had been well determined by aircraft and dropsonde observations. It had been known for some time that hurricanes are warm core vortices; the aircraft data showed that much of the horizontal temperature gradient is concentrated in the eye and eyewall and that in the upper troposphere, the eye temperature can be $15 \mathrm{~K}$ warmer than their environment at the same pressure. Analyses of the entropy distribution (Figure 1) tended to confirm the Riehl-Kleinschmidt-Malkus view of the energy cycle, with a pronounced inward increase of equivalent potential temperature near the storm's eyewall. These observations made it clear that there is a strong surface entropy source under the eyewall.

At about the same time, Ooyama (1969) published the results of the first successful numerical simulation of a tropical cyclone, showing among other things that intensification of such storms indeed relies crucially on surface enthalpy fluxes. A decade later, Rosenthal published the results of a numerical simulation in which he had 
accidentally omitted the cumulus parameterization; the simulated storm had no difficulty intensifying into a mature tropical cyclone (Rosenthal, 1978). Influenced by the Rosenthal and Ooyama results, Douglas Lilly started work on a steady state model based on conservation of certain key quantities along streamlines emanating from the boundary layer. As he was not satisfied with certain properties of his model, Lilly put this work aside until 1984 when he learned of research on the same subject being carried out by the author and Richard Rotunno. The three of us conducted a lively correspondence over the following year, with the intention of publishing our results in two or three papers. Although we wrote two conference preprint papers together (Emanuel et al., 1985; Lilly and Emanuel, 1985) and my own work was written up (Emanuel, 1986), Lilly never formally published his own work on the steady-state hurricane model. As there are some interesting features of this work and because it departs in certain substantial ways from Emanuel (1986), it is worth reviewing here.

HURRICANE INEZ

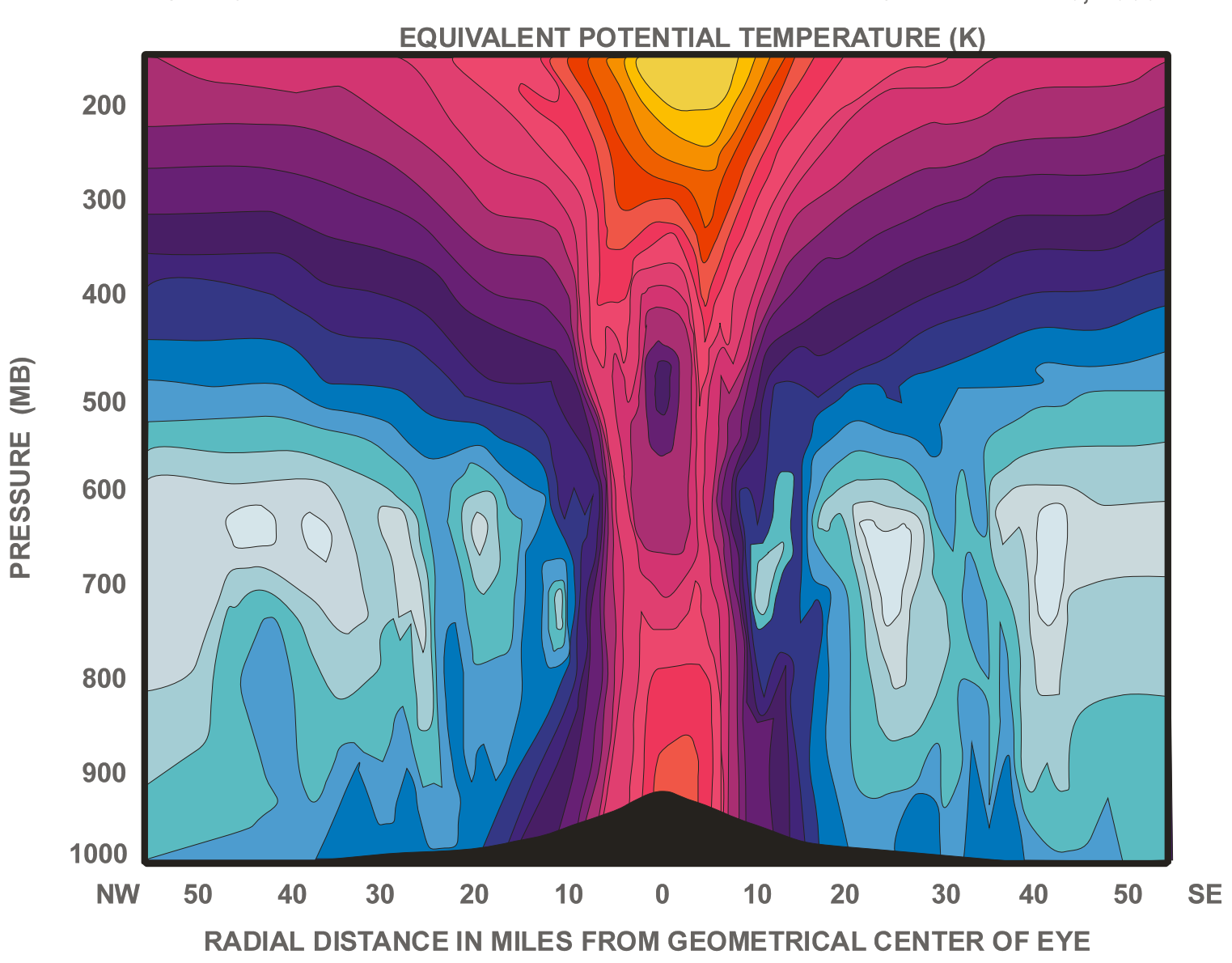

Figure 1: Equivalent potential temperature $(K)$ as a function of pressure and radius from the center of Hurricane Inez on 28 September, 1966, based on aircraft data at $500 \mathrm{~m}, 750$

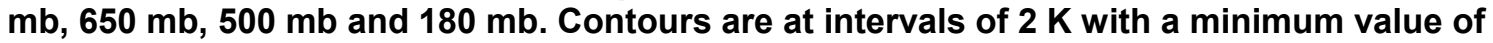
$336 \mathrm{~K}$ (light blue) and a maximum value of $376 \mathrm{~K}$ (yellow). After Hawkins and Imbembo (1976). 
Assuming a steady, circularly symmetric vortex with reversible adiabatic flow above the boundary layer, Lilly first derived the differential relationship

$$
T d s+\frac{M}{r^{2}} d M-\frac{1}{\rho r} \xi d \psi=d\left[E+\frac{1}{2} f M\right],
$$

where $M$ is the angular momentum per unit mass, $s$ is the specific (moist) entropy, $\xi$ is the azimuthal component of the vorticity, $\psi$ is the mass streamfunction, $f$ is the Coriolis parameter (assumed constant over the diameter of the storm), $r$ is the radius from the storm center, $\rho$ is the air density and $E$ is the energy content per unit mass, defined

$$
E \equiv \frac{1}{2}|\mathbf{V}|^{2}+C_{p} T+L_{v} q+g z
$$

where $\mathbf{V}$ is the three-dimensional velocity vector, $C_{p}$ is the heat capacity at constant pressure, $L_{v}$ is the latent heat of vaporization, $q$ is the specific humidity, $g$ is the acceleration of gravity and $z$ is the altitude.

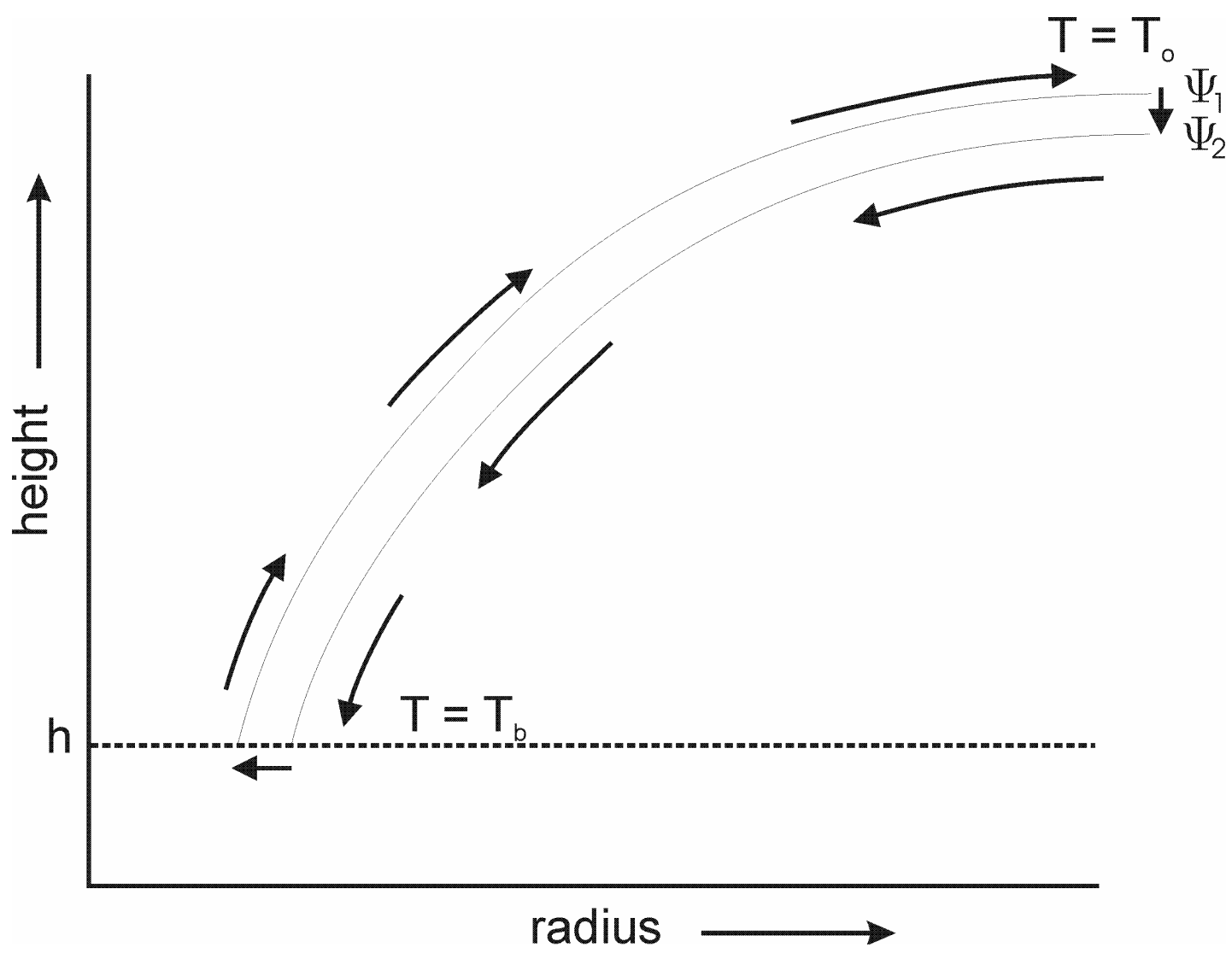

Figure 2: Showing the path of integration of (14) around a closed circuit consisting of a pair of adjacent streamlines emanating from a boundary layer of depth $h$. 
Lilly pointed out that the last term on the left side of (14) vanishes if hydrostatic and gradient wind balance are assumed. Ignoring that term and integrating around a closed circuit consisting of adjacent streamlines, as illustrated in Figure 2, gives

$$
\frac{1}{r_{b}^{2}}=\frac{1}{r_{o}^{2}}-2\left(T_{b}-T_{o}\right) \frac{d s}{d M^{2}}
$$

where $r_{b}$ is the radius of at the top of the boundary layer, $r_{o}$ is the radius that the streamlines approach far from the storm, and $T_{b}$ and $T_{o}$ are the absolute temperatures at the top of the boundary layer and near the top of the storm, respectively.

The expression (16) was derived on quite different grounds by Kleinschmidt (1951) and later by Emanuel (1986). They assumed hydrostatic and gradient wind balance from the start, and simply integrated the thermal wind equation upward along angular momentum surfaces assuming that the saturation entropy, $s^{*}$, is constant on angular momentum surfaces. Kleinschmidt argued, as did Lilly, that air ascending in the core would be saturated and would preserve its values of both entropy and angular momentum as it ascended; thus entropy would be invariant along angular momentum surfaces. I used a different (and I think more general) argument: Even outside the core, where the air is not saturated on the vortex scale, slantwise moist convection should adjust the saturation entropy to be constant on angular momentum surfaces; this is just the condition for neutrality to slantwise convection. This condition of slantwise neutrality has been well verified in simulations using a nonhydrostatic model (Rotunno and Emanuel, 1987). Thus a more general form of (16) is

$$
\frac{1}{r_{b}^{2}}=\frac{1}{r_{o}^{2}}-2\left(T_{b}-T_{o}\right) \frac{d s^{*}}{d M^{2}} .
$$

We note here that Lilly's approach has the advantage that neither hydrostatic nor gradient wind balance has to be assumed; the approach of Kleinschmidt and Emanuel has the advantage that there is no need to invoke energy conservation or to assume that streamlines are along angular momentum surfaces. Thus the approach based on thermal wind balance is equally applicable to a nonsteady vortex, as long as the evolution of the vortex is slow enough that thermal wind balance still applies. I argue that in contrast to (16), the relation (17) is valid everywhere that moist convection occurs; in a mature hurricane, this is most everywhere, except in the eye. Shutts (1981) also derived an expression similar to (16), except that he assumed that dry entropy (potential temperature) rather than moist entropy or saturation moist entropy is invariant on angular momentum surfaces.

The relation (17) strongly constrains the structure of the hurricane vortex, a fact we shall exploit in the next section. When coupled with relations governing sources and sinks of entropy and angular momentum, this relation also places strong constraints on the maximum wind speed of the hurricane. To demonstrate this, we first put (16) in a form that makes explicit its reliance on entropy and angular momentum sources. Assuming that the radii $r_{o}$ to which angular momentum surfaces flair near the top of the 
storm are very much larger than the radii $\left(r_{b}\right)$ that they have at the top of the boundary layer, we can express (16) as

$$
\left(T_{b}-T_{o}\right) \frac{d s}{d t}+\frac{M}{r^{2}} \frac{d M}{d t}=0
$$

in which it is understood that we shall be evaluating the sources of entropy and angular momentum at the top of the boundary layer. Lilly took the top of the boundary layer to be the top of the shallow convective layer, near the level where entropy (equivalent potential temperature) reaches a minimum value. In practice, this is 3-4 $\mathrm{km}$ above the surface. I prefer to take the top of the boundary layer to be the top of the well mixed, subcloud layer, around $500 \mathrm{~m}$ above the surface (Emanuel, 1986). In either case, the equations for the total derivatives of entropy and angular momentum, integrated through a boundary layer of depth $h$, may be written

$$
h \frac{d \bar{s}}{d t}=\frac{1}{T_{s}}\left[C_{k}|\mathbf{V}|\left(k_{s}^{*}-k\right)+C_{D}|\mathbf{V}|^{3}+F_{b}\right]
$$

and

$$
h \frac{d \bar{M}}{d t}=-C_{D} r|\mathbf{V}| V,
$$

where $\bar{S}$ and $\bar{M}$ are the entropy and angular momentum averaged through the depth of the boundary layer, $|\mathbf{V}|$ is a near-surface wind speed, $V$ is the azimuthal velocity of air near the surface, $C_{k}$ and $C_{D}$ are surface exchange coefficients for enthalpy and momentum, respectively, $k_{s}^{*}$ and $k$ are the specific enthalpies of air at saturation at sea surface temperature and pressure, and boundary layer air, respectively, and $F_{b}$ is the enthalpy flux through the top of the boundary layer. We have used the classical bulk formulae for the surface fluxes of enthalpy and momentum, and assumed that there is little turbulent flux of angular momentum through the top of the boundary layer, owing to the very weak vertical gradients of angular momentum found at lower levels in hurricanes. The first term on the right side of (19) is the surface enthalpy flux; the second term is the entropy source owing to dissipative heating, and the final term is the entropy source (usually a sink) owing to enthalpy fluxes through the top of the boundary layer. Both Lilly and Emanuel neglected to include the dissipative heating term, which Bister and Emanuel (1998) later found to be of first order importance.

Lilly's approach, taking the boundary layer depth to be that of the shallow cumulus layer, has the advantage that it is plausible to assume that the enthalpy flux through the top of the boundary layer, $F_{b}$, vanishes. On the other hand, since moist entropy itself varies significantly with altitude within this layer, the relationship between $\bar{s}$ and the saturation entropy at the top of the boundary layer is problematic. In my approach, taking the boundary layer to be the well-mixed subcloud layer, the entropy should be well mixed in the vertical, while convective neutrality would argue that $\bar{s}$ should be nearly equal to the saturation entropy above the top of the boundary layer; on 
the other hand, $F_{b}$ will usually be significant. In the eyewall of a well-developed storm, however, both the vertical entropy gradient in the boundary layer and $F_{b}$ should be very small in the eyewall. Thus, in the eyewall, we may assume that $F_{b} \approx 0$ and $\bar{s} \approx s$. Also approximating $M$ by $r V$ in this region and taking $|\mathbf{V}| \cong V$ allows one to derive, by substituting (19) and (20) into (18), the relation

$$
v_{\max }^{2}=\frac{T_{s}-T_{o}}{T_{o}} \frac{C_{k}}{C_{D}}\left(k_{s}^{*}-k\right),
$$

in which we have assumed that the wind speed so computed represents an upper bound, given that we have neglected enthalpy fluxes through the top of the boundary layer, which are almost always negative. The expression derived by both Lilly and Emanuel (1986) differs from (21) in that $T_{s}$ rather than $T_{o}$ appears in the denominator, as a consequence of neglecting dissipative heating. I interpreted (21) in terms of a Carnot cycle, in which enthalpy is added to the system at the high temperature of the ocean and removed at the low temperature of the storm's outflow near the tropopause.

Equation (21) is in many respects similar to (13) from Riehl (1963), with the same dependence on the ratio of the exchange coefficients and the ambient thermodynamic disequilibrium between the ocean and atmosphere (though here expressed in terms of enthalpy rather than entropy). But there are two differences: unlike (13), (21) has no explicit dependence on outer radius, radius of maximum wind, or wind speed at some particular radius; and Riehl's factor of 100 is replaced by a modified thermodynamic efficiency. Riehl's assumption that parcels become neutrally buoyant at $100 \mathrm{mb}$ has been replaced by an explicit dependence on outflow temperature, which depends on the level of neutral buoyancy of air ascending in the eyewall. Also, Riehl's use of a power law dependence of wind on radius has been replaced by the assumption of thermal wind balance and slantwise neutrality (or, equivalently, of an assumption of energy equilibrium); this gets rid of the factor in brackets in (13).

The relation (21) suggests a strong sensitivity of hurricane intensity to those boundary layer processes that determine the exchange of enthalpy and momentum with the ocean, and ocean temperature near the eyewall, which can strongly affect $k_{s}{ }^{*}-k$.

It is sometimes remarked that (21) is especially sensitive to assumptions about the value of the enthalpy $(k)$ under the eyewall (Holland, 1997). But $k$ is not a free parameter. According to the subcloud layer equilibrium hypothesis (Raymond, 1995), air in the boundary layer is very nearly neutral to adiabatic displacement to a position just above the top of the boundary layer. This may be expressed as $k=h_{b}^{*}$, where $h_{z b}^{*}$ is the saturated moist static energy just above the top of the boundary layer. But $h_{z b}^{*}$ is not arbitrary: through the thermal wind relation (17) it has a specific relationship to the unperturbed saturation moist static energy of the environment. Since angular momentum increases outward, the saturation entropy (and the saturation moist static energy) must increase inward, so that $h_{z b}^{*}$ (and therefore $k$ ) is greater than the value it has in the unperturbed environment. In (21), this offsets the inward increase in $k_{s}^{*}$ that arises from decreasing surface pressure. These effects are quantified in the Appendix. Emanuel 
$(1986,1995)$ simplified the calculation of $k$ at the radius of maximum winds by assuming that the boundary layer relative humidity is constant outside the radius of maximum winds.

The predictions of (21) are in good accord with numerical experiments, beginning with those by Ooyama (1969) and Rosenthal (1971) and continuing with many others in the 1990s, in which the exchange coefficients are simply specified. Unfortunately, little is known about how these coefficients behave at high wind speeds in nature. As is apparent in Figure 1, most of the entropy increase in the inflow occurs very near the eyewall; it is here that hurricanes are sensitive to the exchange coefficients. (Note that for this reason, the centers of hurricanes can approach very near to land before their intensity begins to diminish.) Measurements at low to moderate wind speeds suggest that the drag coefficient increases with wind speed, because of increased surface roughness, but the enthalpy exchange coefficient remains approximately constant (Large and Pond, 1982); when extrapolated to hurricane wind speeds, this would yield a ratio $C_{k} / C_{D}$ too small to explain the observed intensity of hurricanes (Emanuel, 1995). This suggests that other physical processes must come into play to enhance the enthalpy exchange and/or diminish drag. Andreas and Emanuel, (1999) suggested that the relevant mechanism is re-entrant sea spray, which transfers significant amounts of enthalpy to the air. Recent estimates of the exchange coefficients from wind-wave tank measurements (Alamaro et al., 2003), from measurements of the ocean current response to tropical cyclones (Shay, 1999) and from wind profiles measured using dropwindsondes (Powell et al., 2003) suggests that their ratio in high winds is not too different from unity. A field experiment that is scheduled to take place in the summer of 2003 will also attempt to make measurements that could help understand the behavior of surface exchanges at extreme wind speeds.

The calculation of $T_{o}$ is straightforward in principle. Since tropical cyclones are subcritical vortices - internal waves can propagate inward against the outflow at upper levels - the outflow temperature represents that environmental temperature to which the entropy surface arising at the radius of maximum winds asymptotes at large radius. It can be calculated given an environmental temperature sounding. The saturation entropy of the ocean surface, $k_{s}{ }^{*}$, is a function of surface pressure as well as ocean temperature and must be calculated iteratively, using a second relationship between pressure and wind speed. This is discussed in Emanuel (1986) and Emanuel (1995). Finally, the actual enthalpy of the boundary layer air, $k$, must be estimated using a boundary layer model or by making an assumption about its radial distribution outside the radius of maximum winds.

In spite of these limitations, calculations of the maximum wind speed made using (21) are in good agreement with those attained in numerical simulations, reviewed above, in which the ocean temperature is fixed and for which, of course, the exchange coefficients are known since they are specified. Real hurricanes are never observed to exceed the limit given by (21) with $C_{k} / C_{D}=1$, but the vast majority fall well short of this limit (Emanuel, 2000). This is probably due in part to the fact that the ocean temperature cools as hurricane pass over, owing to strong upward mixing of cold water, but also to disruption of the energy cycle by atmospheric interactions which serve, among other things, to import low entropy air into the storm's core. 
The sensitivity of (21) to local perturbations of sea surface temperature can be seen by noting that under average tropical conditions, a local decrease of sea surface temperature of only $2.5^{\circ} \mathrm{C}$ suffices to bring $k_{s}^{*}-k$ to zero. (But note that large-scale gradients of sea surface temperature are associated with similar gradients in $k$, so that $k_{s}^{*}-k$ may remain approximately constant over large areas of undisturbed ocean.) This would suggest that the observed ocean cooling of order $1^{\circ} \mathrm{C}$ under the storm core could have a significant feedback on hurricane intensity. But the first simulation of a hurricane using an coupled ocean-atmosphere model, by Chang and Anthes (1979), showed little effect of the ocean feedback on storm intensity, leading to a period of roughly two decades during which ocean feedback was regarded as unimportant, except perhaps for storms crossing the wakes of previous storms. (In hindsight, the model used by Chang and Anthes had too coarse a resolution and was integrated for too short a period to see appreciable effects from ocean feedback.) Interest in ocean feedback was renewed after publication of Sutyrin and Khain (1984), Gallacher et al. (1989), Khain and Ginis (1991), Bender et al. (1993), and Schade and Emanuel (1999), all of whom used advanced coupled models to demonstrate that ocean feedback has a first-order effect on hurricane intensity. Emanuel (1999) demonstrated that the intensity of many hurricanes could be accurately predicted using even a very simple atmospheric model coupled to an essentially one-dimensional ocean model (Schade, 1997), as long as storms remain unmolested by adverse atmospheric influences such as environmental wind shear, which has been shown to be a statistically significant predictor of intensity change.

\section{Physical constraints on hurricane structure}

The derivation of (21) relies on the assumptions that the boundary layer entropy is equal to the saturation entropy above the boundary layer (i.e. convective neutrality), that the angular momentum is dominated by $r V$ and that we can neglect the turbulent flux of enthalpy through the top of the boundary layer at the radius of maximum winds. If these assumptions truly applied everywhere, then (21) would be valid everywhere; clearly this is not the case as the right side of (21) has only a very weak dependence on radius. Here we argue that the main features that determine the radial structure of the hurricane vortex are radial variations in the enthalpy flux through the top of the boundary layer $\left(F_{b}\right)$, and, in the far outer region, the decoupling of the boundary layer from the free troposphere in regions that are stable to convection.

Observations (e.g. Powell, 1990a,b) and numerical simulations (e.g. Rotunno and Emanuel, 1987) reveal that the main mechanisms for evacuating enthalpy from the boundary layer are low entropy convective downdrafts and turbulent entrainment. In the spirit of simplicity, we represent these processes using a simple convective scheme based on Raymond's (1995) boundary layer quasi-equilibrium hypothesis. This scheme is described in detail in Emanuel (1995). As shown in Figure 3, we represent convective updraft volume flux by $M_{u}$, convective downdraft volume flux by $M_{d}$, clear-air vertical velocity by $w_{e}$, and total vertical velocity by $w$. For convenience, we define $M_{d}$ and $w_{e}$ to be positive downward. The flux of low enthalpy air into the top of the boundary layer is then just 


$$
F_{b}=-\left(w_{e}+M_{d}\right)\left(h_{b}-h_{m}\right)
$$

where $h_{b}$ and $h_{m}$ are the moist static energies of air in the boundary layer and just above the top of the boundary layer, respectively. We have assumed that both convective downdrafts and clear-air descent advect the same characteristic value of moist static energy into the boundary layer, and that $w_{e}>0$, i.e. that the clear air is actually sinking.

At the same time, mass continuity demands that

$$
M_{u}-M_{d}-w_{e}=w
$$

i.e. that the three components add up to the total vertical velocity. Using (23) in (22) gives

$$
F_{b}=-\left(M_{u}-w\right)\left(h_{b}-h_{m}\right) .
$$

Using this in the boundary layer entropy equation (19), and expanding the total derivative of entropy in angular momentum coordinates:

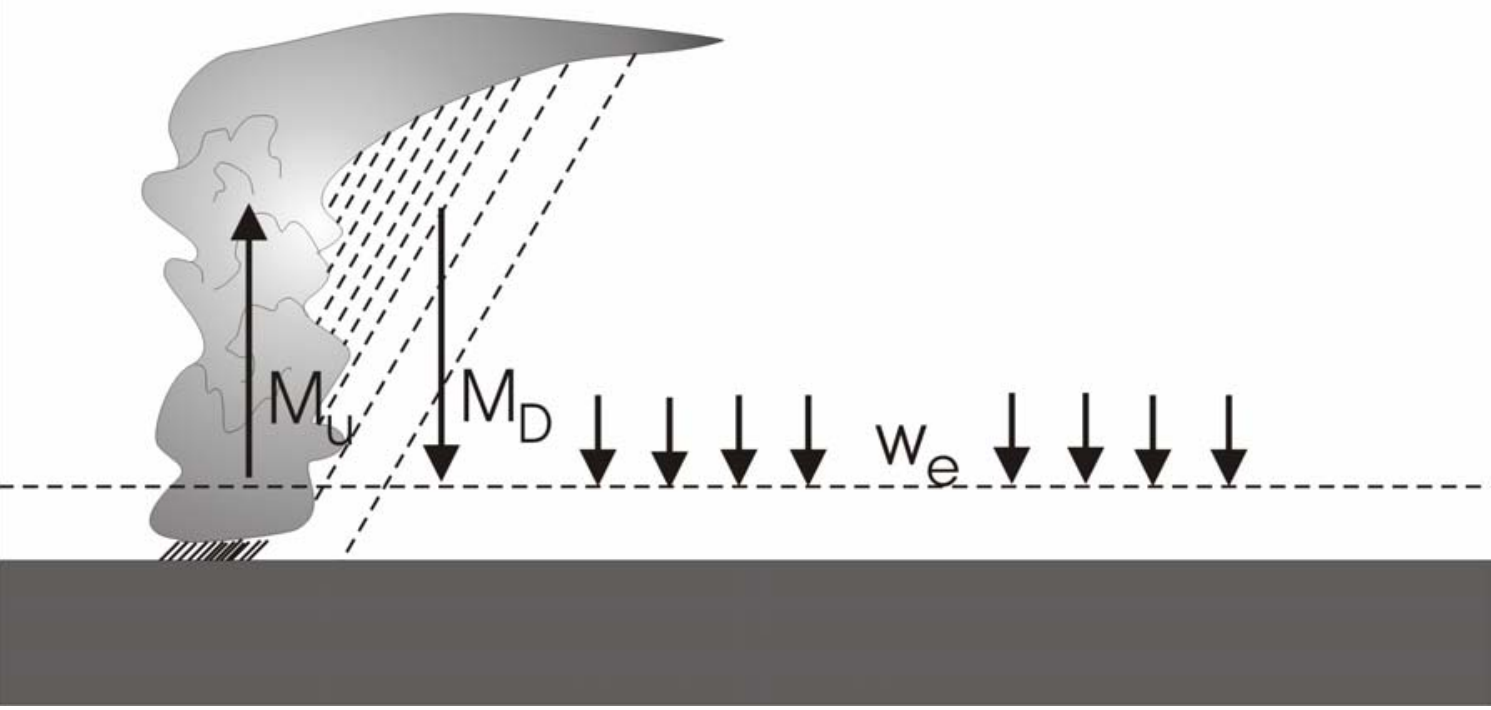

Figure 3: Partition of vertical motions at the top of the subcloud layer into convective updrafts, convective downdrafts, and clear-air subsidence.

allows us to write (19) as

$$
\frac{d s}{d t}=\frac{\partial s}{\partial \tau}+\frac{d M}{d t} \frac{\partial s}{\partial M}
$$




$$
h T_{s} \frac{\partial s}{\partial \tau}=C_{k}|\mathbf{V}|\left(k_{s}^{*}-k\right)+C_{D}|\mathbf{V}|^{3}-\left(M_{u}-w\right)\left(h_{b}-h_{m}\right)-h T_{s} \frac{d M}{d t} \frac{\partial s}{\partial M}
$$

Finally using (20) for the boundary layer sink of angular momentum in (25) gives

$$
h T_{s} \frac{\partial s}{\partial \tau}=C_{k}|\mathbf{V}|\left(k_{s}^{*}-k\right)+C_{D}|\mathbf{V}|^{3}-\left(M_{u}-w\right)\left(h_{b}-h_{m}\right)+T_{s} C_{D} r|\mathbf{V}| V \frac{\partial s}{\partial M}
$$

Note that we have assumed here that both entropy and angular momentum are well mixed in the vertical within the boundary layer.

We are going to use (26) in two different ways, depending on whether convection is present or absent. Where convection is absent, it is assumed that the boundary layer entropy is decoupled from the saturation entropy aloft. We can then use (26), with $M_{u}=0$, to calculate the radial distribution of entropy in the steady state. But there is little incentive to actually carry out the calculation, since the boundary layer entropy will then have no control over the vortex structure as a whole.

Where convection is present, we invoke boundary layer quasi-equilibrium, which sets the left side of (26) to zero, and use it as a closure for $M_{u}$. We also assume that the saturation entropy above the boundary layer, $s *$ is equal to the boundary layer entropy, $s$, when convection is active. Then, with the help of the thermal wind balance (17) and once again neglecting $1 / r_{o}^{2}$, we can write the last term of (26) as

$$
T_{s} C_{D} r|\mathbf{V}| V \frac{\partial s}{\partial M} \simeq-C_{D}|\mathbf{V}| V \frac{T_{s}}{T_{s}-T_{o}} \frac{M}{r} .
$$

Approximating $M / r$ by $V$, and $|\mathbf{V}|$ also by $V$, and using this in (26) gives the closure for the convective updraft mass flux:

$$
M_{u}=w+\frac{1}{h_{b}-h_{m}}\left[C_{k} V\left(k_{s}^{*}-k\right)-C_{D} V^{3} \frac{T_{o}}{T_{s}-T_{o}}\right] .
$$

Using boundary layer quasi-equilibrium has allowed us to close on the convective mass flux, but in the process we have lost the prediction of boundary layer entropy. The missing ingredient is the thermodynamic balance above the boundary layer. Along an angular momentum surface (also a surface of constant $s^{*}$ by the assumption of slantwise convective neutrality), the temperature (equivalently $s^{*}$ ) is controlled by convection and radiation:

$$
\frac{\partial s *}{\partial \tau}=\frac{\Gamma_{d}}{\Gamma_{m}}\left[\left(M_{u}-M_{d}-w\right) \frac{\partial s_{d}}{\partial z}+\frac{\dot{Q}_{r a d}}{T}\right],
$$


where $\Gamma_{d}$ and $\Gamma_{m}$ are the dry and moist adiabatic lapse rates, $s_{d}$ is the entropy of dry air, and $\dot{Q}_{\text {rad }}$ is the radiative heating. We relate the downdraft mass flux to the updraft mass flux by

$$
M_{d}=(1-\varepsilon) M_{u},
$$

where $\varepsilon$ is a bulk precipitation efficiency. When it is unity, there is no downdraft, while when it is zero the updraft and downdraft mass fluxes are equal. Using this in (28) and assuming a steady state gives

$$
w=-w_{\text {rad }}+\varepsilon M_{u}
$$

where

$$
w_{\text {rad }} \equiv-\dot{Q}_{r a d} / T \frac{\partial s_{d}}{\partial z}
$$

Since the tropical troposphere is usually cooling radiatively, $w_{\text {rad }}$ is usually positive. It is the rate at which air subsides in the troposphere under the influence of radiative cooling. In this simple model, we shall just take it to be a constant.

In summary, in nonconvective regions in which (27) gives a zero or negative value for the convective updraft mass flux, we have, from (30), that

$$
w=\frac{1}{r} \frac{\partial \psi}{\partial r}=-w_{\text {rad }} \quad \text { when } M_{u}=0
$$

where $\psi$ is the streamfunction at the top of the boundary layer. But where convection is active (i.e. (27) yields a positive convective updraft mass flux), substitution of (27) into (30) gives

$$
(1-\varepsilon) \frac{1}{r} \frac{\partial \psi}{\partial r}=-w_{r a d}+\frac{\varepsilon}{h_{b}-h_{m}}\left(C_{k} V\left(k_{s}^{*}-k\right)-C_{D} V^{3} \frac{T_{o}}{T_{s}-T_{o}}\right), \text { when } M_{u}>0
$$

To close the system, we use the steady-state form of the boundary layer angular momentum equation, (20):

$$
\psi \frac{\partial M}{\partial r}=C_{D} r^{2} V^{2}
$$

or equivalently,

$$
\frac{\partial(r V)}{\partial r}=\frac{C_{D} r^{2} V^{2}}{\psi}-f r
$$


Thus the closed steady-state system consists of (33) together with either (31) or (32), depending on the sign of $M_{u}$ determined from (27).

This system of equations is appropriate to the outer region of the storm and to the outer part of its eyewall, where we expect a match between the vertical motion in the free troposphere an that demanded by Ekman dynamics at the top of the boundary layer. However, it is not applicable at the inner edge of the eyewall, where radial diffusion is necessary to balance to strong frontogenetical tendencies, or in the eyewall where Ekman pumping produces upflow only through a shallow layer, while inward turbulent fluxes of angular momentum drive an axial downflow above the boundary layer (Emanuel, 1997). Thus we terminate integration of the equations near the radius of maximum winds and do not use them to derive a maximum wind speed.

We next proceed to a simple numerical solution of (33) with either (31) or (32). Before doing this, we can absorb most of the parameter dependence of these equations into scaling of the dependent and independent variables. We replace the variables as follows:

$$
\begin{gathered}
V \rightarrow v_{\max } V, \\
r \rightarrow \frac{v_{\max }}{f} r, \\
\psi \rightarrow C_{D} \frac{v_{\max }^{3}}{f^{2}} \psi, \\
w_{\text {rad }} \rightarrow C_{D} v_{\max } w_{Q}, \\
M_{u} \rightarrow C_{D} v_{\max } M_{u},
\end{gathered}
$$

where $v_{\max }$ is defined by (21). With these substitutions, our system becomes

$$
\begin{gathered}
\frac{\partial(r V)}{\partial r}=\frac{r^{2} V^{2}}{\psi}-r, \\
\frac{1}{r} \frac{\partial \psi}{\partial r}=-w_{Q} \quad \text { for } M_{u}=0, \\
\frac{1-\varepsilon}{r} \frac{\partial \psi}{\partial r}=-w_{Q}+\varepsilon \Lambda\left(V-V^{3}\right) \quad \text { for } M_{u}>0,
\end{gathered}
$$

with

$$
M_{u}=\frac{1}{1-\varepsilon}\left[-w_{Q}+\Lambda\left(V-V^{3}\right)\right]
$$

Here the additional nondimensional parameter is defined 


$$
\Lambda \equiv \frac{C_{k}}{C_{D}} \frac{k_{s}^{*}-k}{h_{b}-h_{m}}
$$

Although $\Lambda$ must vary with radius, since all of its components do, we shall take it to be a constant here for simplicity; likewise, we shall neglect radial variations of the precipitation efficiency, $\varepsilon$.

The boundary condition for this system is that $\psi$ vanishes at some outer radius $r_{o}$. From (34) $V$ must vanish there as well. Although the system is second order in $r$, we do not apply a second boundary condition since we terminate integration at or outside the radius of maximum winds. The control parameters are then $\Lambda, \varepsilon, w_{Q}$ and $r_{o}$.

Before turning to numerical integrations, it is instructive to look at approximate analytic solutions in the far outer region, where it will turn out that $M_{u}=0$. In that case, we can integrate (35) directly, and applying the boundary conditions, we get

$$
\psi=\frac{1}{2} w_{Q}\left(r_{o}^{2}-r^{2}\right)
$$

Substituting this into (34) gives

$$
\frac{\partial(r V)}{\partial r}=\frac{2 r^{2} V^{2}}{w_{Q}\left(r_{o}^{2}-r^{2}\right)}-r .
$$

For $w_{Q}<<1$, the dominant balance of the above gives

$$
V^{2} \approx \frac{1}{2} w_{Q} \frac{r_{o}^{2}-r^{2}}{r} .
$$

Away from the outer radius $r_{o}$, this gives the same $r^{-1 / 2}$ dependence of $V$ derived by (Riehl, 1963) on the somewhat questionable premise that potential vorticity is conserved in the outer region.

Closer in towards the center, but still well outside the radius of maximum winds, another approximate solution presents itself. Here we assume that convection is active, so that both (34) and (36) apply. We also assume that $\varepsilon \Lambda V>>w_{Q}$ but $V^{2}<<1$, so that (36) may be approximated by

$$
\frac{\partial \psi}{\partial r} \simeq \frac{\varepsilon \Lambda}{1-\varepsilon} r V
$$


while at the same time, the last term in (34) may be neglected (i.e. the relative vorticity is much greater than the Coriolis parameter), giving

$$
\frac{\partial}{\partial r}(r V) \simeq \frac{r^{2} V^{2}}{\psi} .
$$

The system comprised of (40) and (41) has the power law solution

where

$$
V \approx r^{-n}
$$

$$
n \equiv \frac{\varepsilon \Lambda-2(1-\varepsilon)}{\varepsilon \Lambda-(1-\varepsilon)} .
$$

Realistic solutions are thus obtainable only if

$$
\Lambda>2 \frac{1-\varepsilon}{\varepsilon} .
$$

Thus the precipitation efficiency has to be relatively large and/or the relative air-sea thermodynamic disequilibrium has to be large. Note that for the parameters used in the numerical solution discussed presently, $n=2 / 3$.

The numerical solution of (34) - (36) is straightforward. We start at $r=r_{o}$ and march inward, using a radial step of 0.001 . A particular solution for the azimuthal wind is shown in Figure 4 and compared to the profile obtained by running the model of Rotunno and Emanuel (1987) into a statistical steady state and re-scaling the velocity and radius to map into the nondimensional coordinates. The agreement is quite good, especially considering the crude approximation of neglecting any radial variations of $\varepsilon$ and $\Lambda$. The longer tail in the Rotunno-Emanuel model is likely owing to the fact that in that model the radiative cooling is proportional to the temperature perturbation rather than being a fixed constant. Thus the dry descent is weaker and must extend over a broader area to carry the same mass flux.

Corresponding solutions for the nondimensional radial and vertical velocities and for the cumulus updraft volume flux are shown in Figure 5 and 6 . Note that convection is absent in the far outer region.

Having obtained asymptotic solutions for the azimuthal velocity for large and small $V$ (but still well outside the radius of maximum winds), we can attempt to patch these together to form a distribution approximately valid for the whole range of radius outside the radius of maximum winds. At the same time, we can build in an asymptotic limit for the wind profile in the eye, to get a distribution for the whole storm. I have attempted to do this while at the same time altering the large $r$ asymptotic limit to better fit the Rotunno and Emanuel numerical solution. The result of this exercise is 


$$
V^{2}=V_{m}^{2}\left(\frac{r_{0}-r}{r_{0}-r_{m}}\right)^{2}\left(\frac{r}{r_{m}}\right)^{2 m}\left[\frac{(1-b)(n+m)}{n+m\left(\frac{r}{r_{m}}\right)^{2(n+m)}}+\frac{b(1+2 m)}{1+2 m\left(\frac{r}{r_{m}}\right)^{2 m+1}}\right],
$$

where $n$ is given by (43), $m$ is an exponent governing the wind profile in the eye, $V_{m}$ is the maximum wind speed, $r_{m}$ is the radius of maximum winds, and $b$ is a weighting parameter that governs the transition between the two asymptotic regimes. Note that (45) is valid as well using dimensional values of the radius, since all the radii are normalized anyway. Also note that unless $r_{0}$ is unreasonably small, the absolute angular momentum implied by (45) will be a monotonically increasing function of radius.

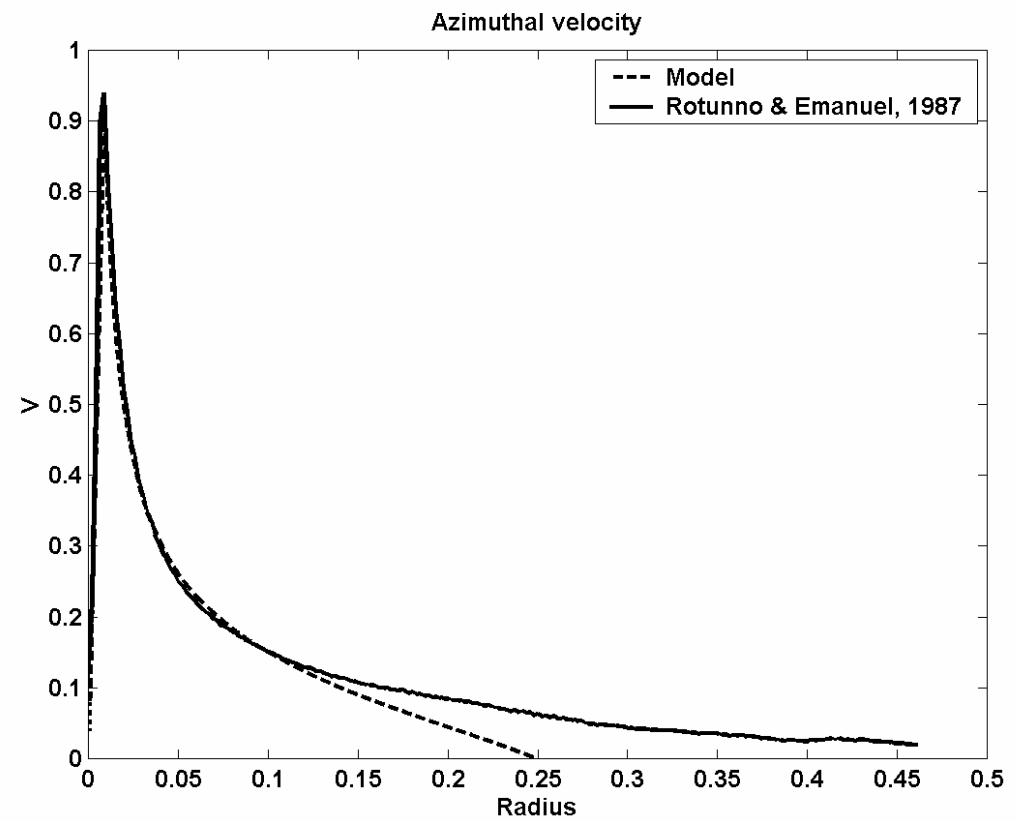

Figure 4: Nondimensional azimuthal velocity as a function of nondimensional radius as a solution of the steady-state model, with $\Lambda=1, \varepsilon=0.8, w_{Q}=0.1$ and $r_{o}=0.25$. For plotting purposes, we let the azimuthal velocity decrease linearly with radius to zero inside the terminal radius of the integration. Shown for comparison in red is the quasi-steady velocity profile from a simulation using the nonhydrostatic model of Rotunno and Emanuel (1987), scaled to these coordinates. 


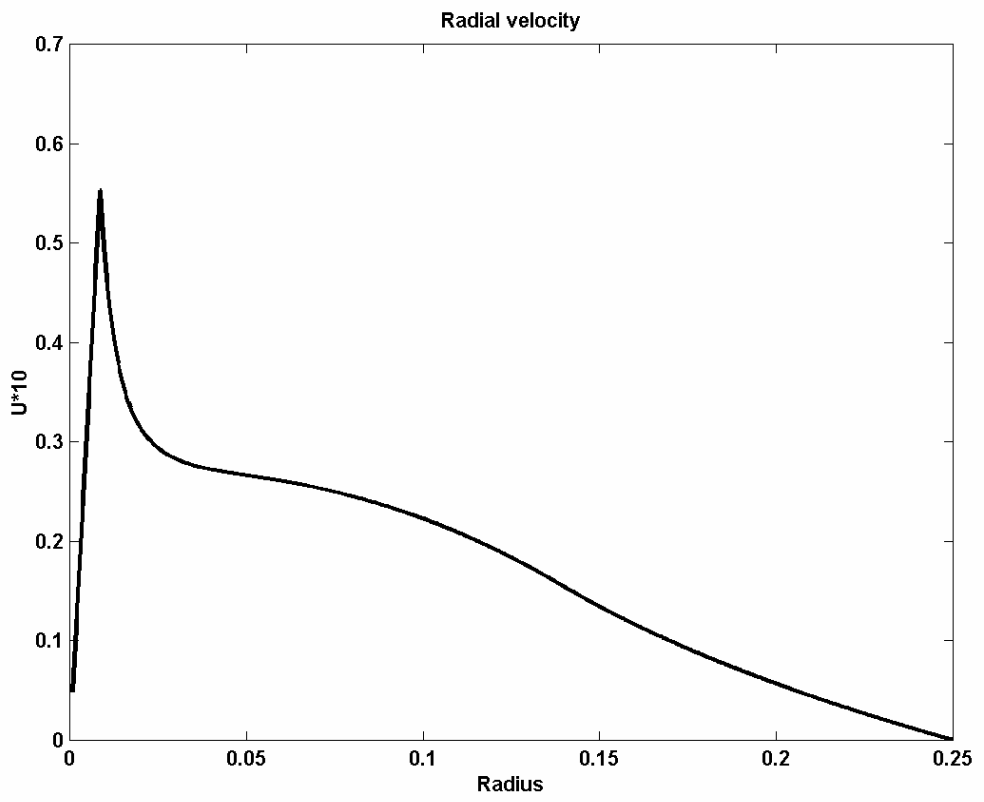

Figure 5: Same as in Figure 4 but showing the nondimensional radial velocity in the boundary layer (with positive values inward).

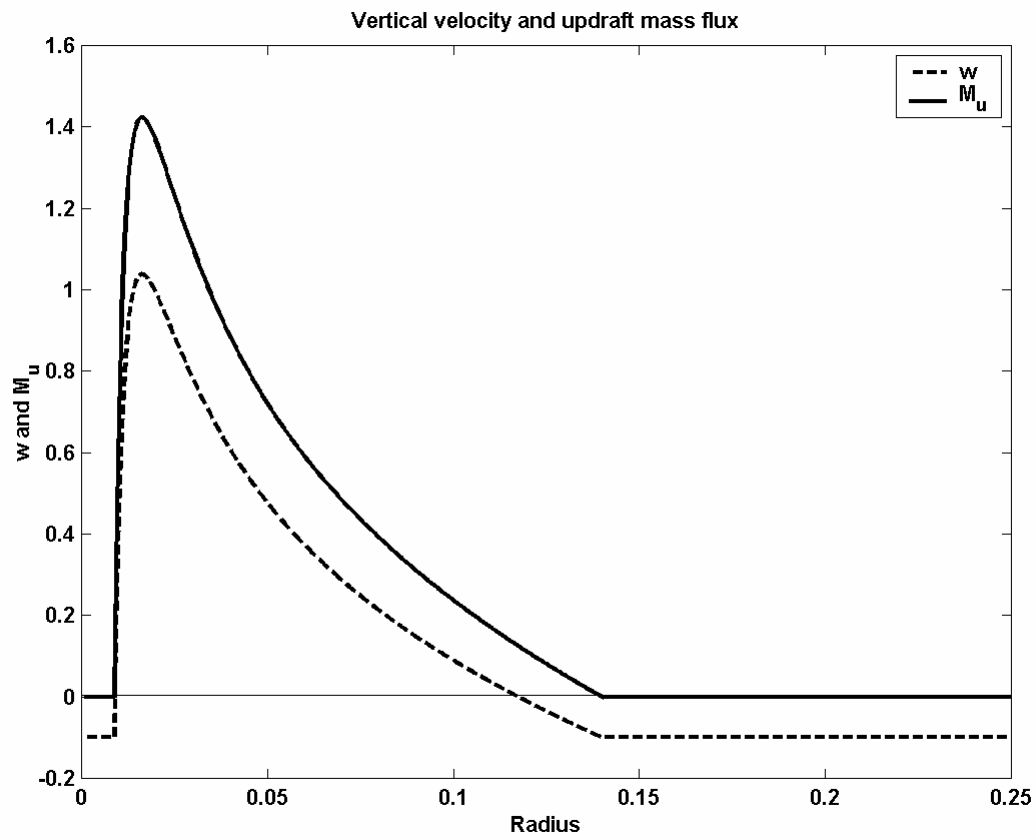

Figure 6: Same as in Figure 4 but showing the total vertical velocity (blue) and the cumulus updraft volume flux (green). 
A solution to (45) taking $V_{m}=74 \mathrm{~m} \mathrm{~s}^{-1}$ and $r_{m}=14 \mathrm{~km}$ from the Rotunno and Emanuel numerical simulation, and using $n=0.9, m=1.6, b=0.25$ and $r_{0}=1200 \mathrm{~km}$ is compared to the R\&E simulation results in Figure 7. The fit is quite good. Also shown is the best fit of Holland's (1980) wind profile, taking his $b$ parameter to be 1.9. The Holland profile is a little too flat in the outer region but quite good in the inner region. It has the advantage, though, of having a simpler form than (45).

Once $V$ has been calculated, the boundary layer streamfunction can be obtained from (34), whence the radial velocity in the boundary layer and the vertical velocity at its top may be derived. These are shown for the same solution in Figures 8 and 9 , respectively. Note that while the vertical velocity is indeed negative in the outer region, it is too small to distinguish from zero in the graph.

We have made one initial attempt to compare (45) with observed wind profiles, using data collected from a NOAA WP-3D aircraft and made available by NOAA's Hurricane Research Division. Figure 10 compares a profile of azimuthal wind at $3 \mathrm{~km}$ altitude from a single radial aircraft pass to the profile in (45) using the same parameters as before, except taking $V_{m}=60 \mathrm{~ms}^{-1}, r_{m}=32 \mathrm{~km}$ and using $n=0.8$. Thus this observed profile is a little flatter than the numerically simulated profile just outside the radius of maximum winds.

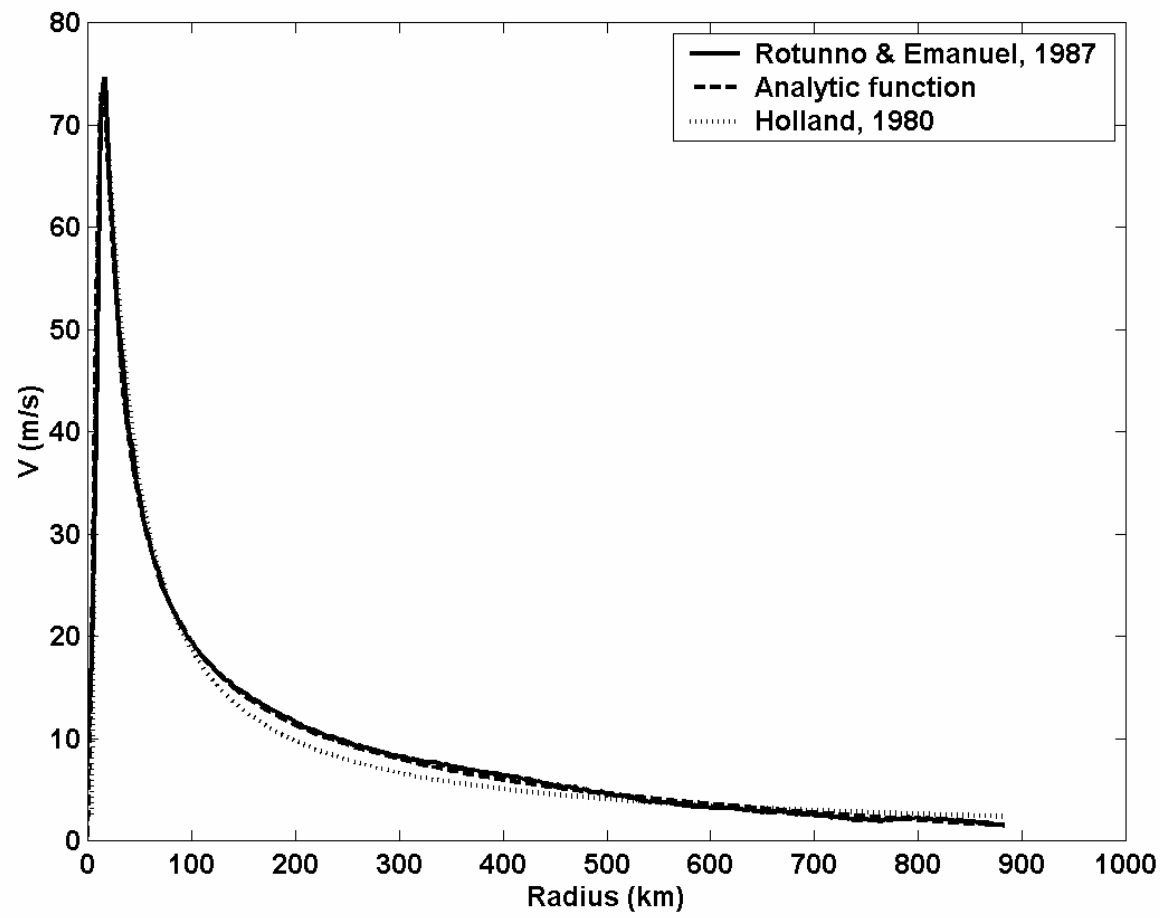

Figure 7: Radial profiles of azimuthal velocity from an integration of the Rotunno and Emanuel (1987) model (solid), from (45) (dashed) and from the analytic model of Holland (1980) (dotted). See text for parameter values used. 


\section{Summary}

Early work on tropical cyclone energetics by Riehl, Kleinschmidt and by Riehl and Malkus recognized that such storms are powered by enthalpy transfer from the ocean. The latter authors came close to developing a correct closed form expression for the maximum sustainable wind speed. Subsequent work by Lilly and by the present author established such an expression, (21), although only in the last decade was the importance of dissipative heating recognized. The energy cycle makes clear that tropical cyclones are highly susceptible to small ocean cooling under their eyewalls, and also emphasizes the importance of the outflow temperature, which is governed by the entropy of the air ascending in the eyewall and the ambient temperature profile.

The intensification of tropical cyclones requires a rapid variation of downdraft enthalpy flux across the eyewall (Emanuel, 1997); this process also determined the radial profile of pressure and wind in this region. The wind profile in the eye itself represents a balance between Ekman pumping, which acts to spin down the circulation

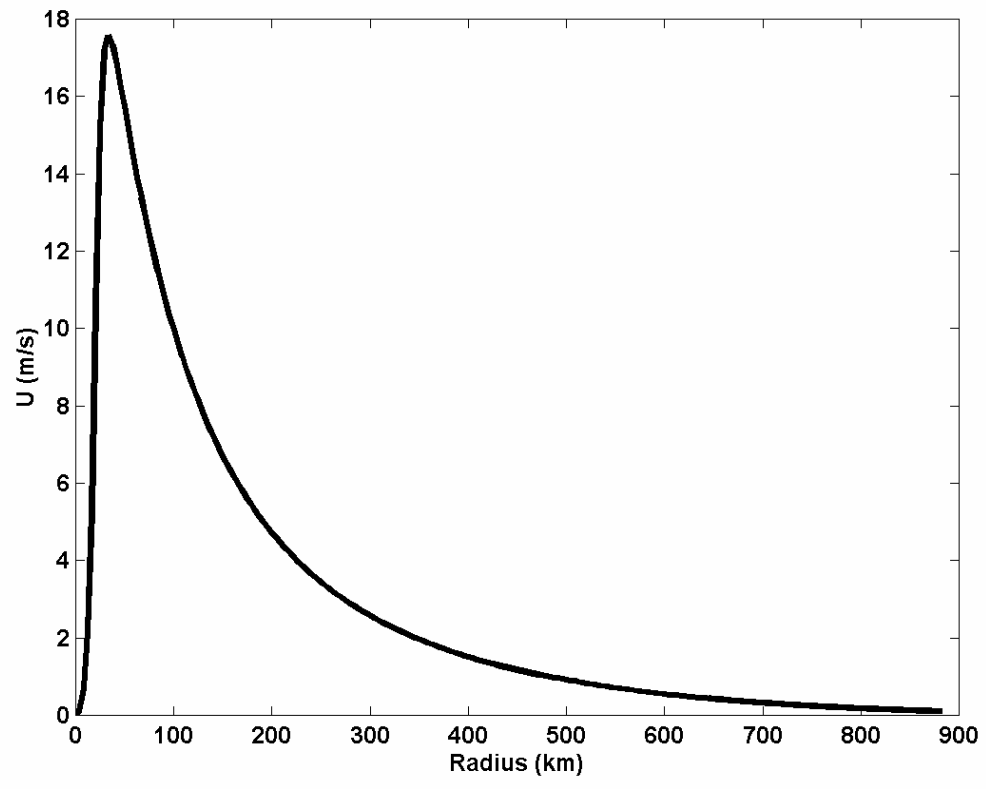

Figure 8: Magnitude of the inward radial velocity derived from (34) using the azimuthal velocity obtained from (45). 


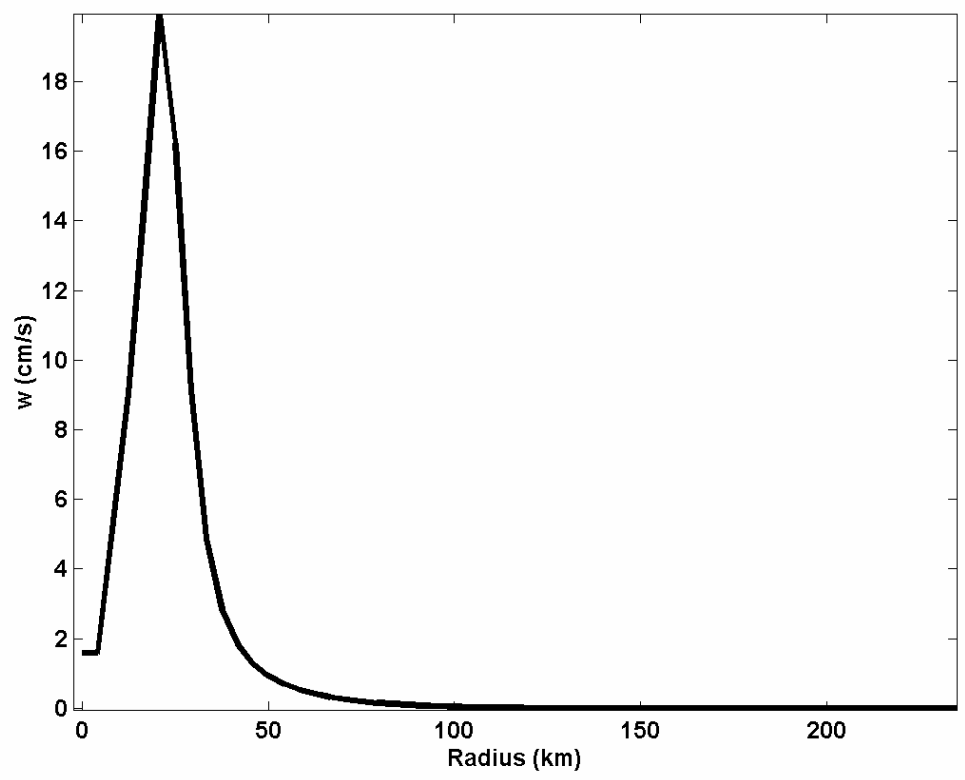

Figure 9: Same as Figure 8 but showing the vertical velocity. Note reduced radial scale.

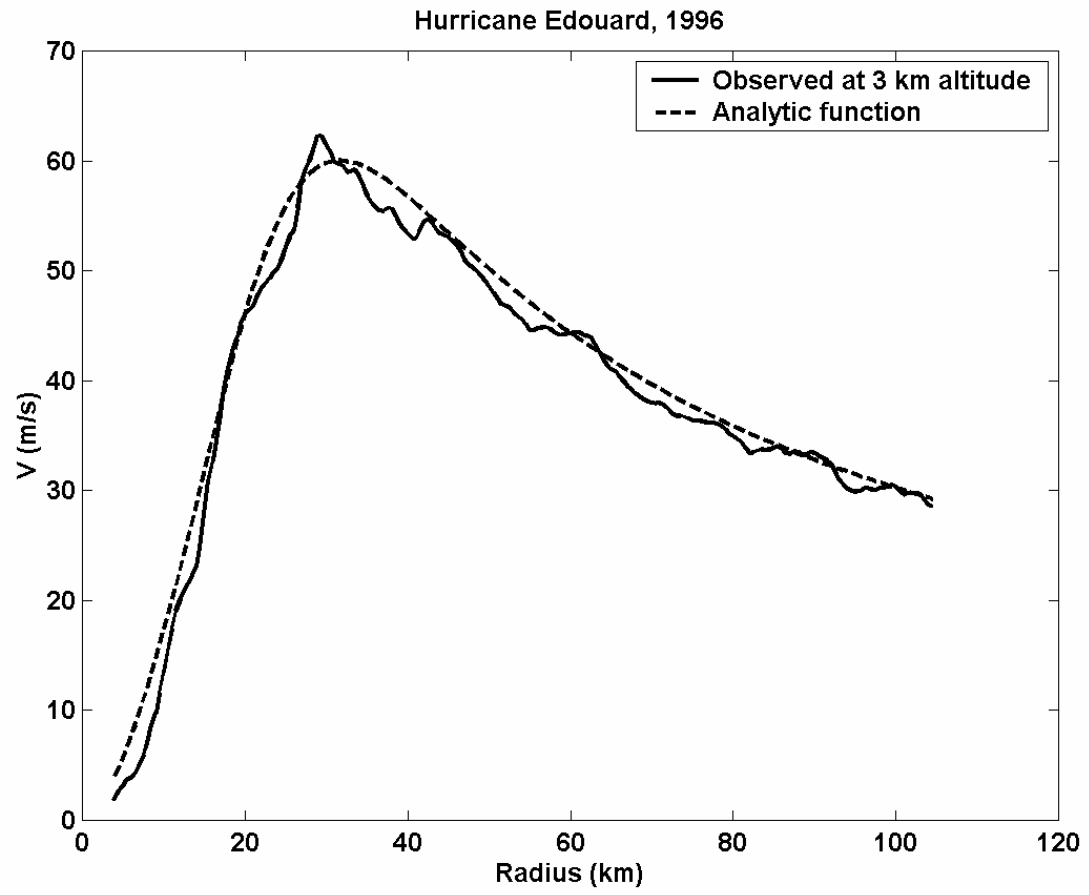

Figure 10: Comparison of (45) to profile of azimuthal wind observed in Hurricane Edouard of 1996. See text for parameter values.

above the boundary layer, and inward turbulent diffusion of angular velocity from the eyewall, which acts in the opposite sense. Outside the eye, the surface wind controls the surface fluxes which, through the boundary layer quasi-equilibrium postulate, control the 
convective flux of enthalpy out the top of the boundary layer. On the other hand, there must be enough upward motion to balance, by adiabatic cooling, the sum of the convective heating and the radiative cooling. But this upward motion must match that demanded by Ekman pumping, which is determined by the radial variation of azimuthal wind. This requirement strongly constrains the radial wind variation outside the core. We here developed a set of nonlinear ODEs that govern this profile and found asymptotic solutions in certain limits. By patching such solutions together, we derived a uniformly valid wind profile, (45), which replicates that found in a numerical simulation using a nonhydrostatic, axisymmetric model. While not as elegant as the simple analytic wind profile proposed by Holland (1980), it does depend explicitly on environmental parameters. In particular, it predicts a steeper decline of wind with radius just outside the core when the mid-level environment is moist, the air-sea thermodynamic disequilibrium is large, and/or the precipitation efficiency is large.

\section{Appendix}

The expression (21) for the maximum wind speed is not closed, because both $k_{s}^{*}$ and $k$ vary with radius. The author (Emanuel, 1986; Emanuel, 1995) developed a closed form expression by assuming that the boundary layer relative humidity under the eyewall is the same as that of the unperturbed environment. Here we point out that the radial variation of both $k_{s}^{*}$ and $k$ depend on the outer vortex structure. First, and without loss of generality, we write (21) in terms of moist static energy rather than enthalpy:

$$
v_{\max }^{2}=\frac{T_{s}-T_{o}}{T_{o}} \frac{C_{k}}{C_{D}}\left(h_{s}^{*}-h\right) .
$$

We next make use of the boundary layer quasi-equilibrium postulate, setting $h$ in (A1) to $h_{b}^{*}$, the saturation moist static energy just above the top of the boundary layer. Variations in $h_{b}^{*}$ at constant altitude are related to variations in the saturation entropy by the first law of thermodynamics:

$$
\delta h_{b}^{*}=T_{b} \delta s^{*}+R_{d} T_{b} \delta \ln p
$$

where $T_{b}$ is the absolute temperature at the top of the boundary layer and we have neglected the difference between total pressure and the partial pressure of dry air. On the other hand, thermal wind balance, as given by (17), relates radial variations of saturation entropy to radial variations of angular momentum. In the limit of very large $r_{o}$, (17) may be written

$$
\left(T_{b}-T_{o}\right) \delta s^{*}=-\frac{M}{r^{2}} \delta M
$$

Now using the definition of angular momentum, (9), and the gradient balance equation, 


$$
R_{d} T \frac{\partial \ln p}{\partial r}=\frac{v^{2}}{r}+f v
$$

we can re-write $(\mathrm{A} 3)$ as

$$
\begin{aligned}
\left(T_{b}-T_{o}\right) \delta s^{*} & =-\left[\delta\left(\frac{1}{2} v^{2}+\frac{1}{2} f r v\right)+\frac{1}{2} f^{2} r \delta r+\left(\frac{v^{2}}{r}+f v\right) \delta r\right] \\
& =-\delta\left[\frac{1}{2} v^{2}+\frac{1}{2} f r v+\frac{1}{4} f^{2} r^{2}+R_{d} T \ln p\right]
\end{aligned}
$$

Substituting (A4) into (A2) and neglecting any radial variations of $T_{b}$ or $T_{o}$, we can integrate the result between the radius of maximum winds at the outer limit of the vortex, where, be definition, $v=0$, to obtain

$$
h_{b}^{*}=h_{a}^{*}-\frac{T_{b}}{T_{b}-T_{o}}\left[\frac{1}{2}\left(v_{\max }^{2}+f r_{m} v_{\max }\right)+R_{d} T_{o} \ln \left(p_{m} / p_{a}\right)-\frac{1}{4} f^{2} r_{o}^{2}\right] \text {, }
$$

where $r_{m}$ is the radius of maximum winds, $r_{o}$ is the outer limit of the vortex, and $p_{m}$ and $p_{a}$ are the surface pressures at the radius of maximum winds and in the unperturbed environment, respectively. Note that boundary layer quasi-equilibrium, applied to the storm environment, gives $h_{a}^{*}=h_{a}$, the boundary layer moist static energy. Also note that gradient wind balance may be used to find $p_{m} / p_{a}$ given $v_{\max }$. This is where the outer wind profile does influence the maximum wind speed, albeit weakly. Here we simplify matters by using an empirical relationship, $R_{d} T_{s} \ln p_{m} / p_{a} \cong-b v_{\max }^{2}$, where $b$ is an empirical constant. Using this, neglecting $f r_{m}$ in comparison to $v_{\max }$ and the difference between $T_{b}$ and $T_{s}$, and substituting (A5) into (A1) gives

$$
v_{\max }^{2} \cong \frac{C_{k}}{C_{D}}\left[\frac{\frac{T_{s}-T_{o}}{T_{o}}\left(h_{s}^{*}-h_{a}\right)-\frac{1}{4} \frac{T_{s}}{T_{o}} f^{2} r_{o}^{2}}{1-\frac{C_{k}}{C_{D}}\left(\frac{1}{2} \frac{T_{s}}{T_{o}}-b\right)}\right] .
$$

Note that there is also a pressure dependence of $h_{s}^{*}$, which we have not accounted for in (A6). The lower pressure at the radius of maximum winds will increase $h_{s}^{*}$ over its ambient value, thus increasing the wind speed over that estimated using the ambient value of the saturation moist static energy of the sea surface. Also note that steeper wind profiles yield smaller values of $b$ and this greater maximum winds. A reasonable value of $b$ is 1 , making the denominator of (A6) slightly large than 1 under typical 
conditions. Finally, as pointed out by Emanuel (1986), the last term in the numerator shows that the maximum wind speed decreases with storm size, through the effect is not large unless the outer radius becomes quite big, of order $1000 \mathrm{~km}$.

\section{References}

Alamaro, M., K. Emanuel and W. McGillis, 2003: Experimental investigation of air-sea transfer of momentum and enthalpy at high wind speed. Bound. Layer Meteor., 109 , submitted

Andreas, E. L. and K. A. Emanuel, 1999: Effects of sea spray on tropical cyclone intensity. Preprints of the 23rd Conf. on Hurricanes and Tropical Meteorology, Dallas, TX, Amer. Meteor. Soc., Boston

Bender, M. A., I. Ginis and Y. Y. Kurihara, 1993: Numerical simulations of tropical cyclone-ocean interaction with a high resolution coupled model. J. Geophys. Res., 98, 23245-23263

Bister, M. and K. A. Emanuel, 1998: Dissipative heating and hurricane intensity. Meteor. Atmos. Physics, 50, 233-240

Byers, H. R., 1944: General Meteorology. McGraw-Hill, New York, 645 pp.

Chang, S. W. and R. A. Anthes, 1979: The mutual response of the tropical cyclone and the ocean. J. Phys. Ocean., 9, 128-135

Charney, J. G. and A. Eliassen, 1964: On the growth of the hurricane depression. J. Atmos. Sci., 21, 68-75

Emanuel, K., R. Rotunno and D. K. Lilly, 1985: An air-sea interaction theory for tropical cyclones. Preprints of the 16th Conference on Hurricanes and Tropical Meteorology, Houston, TX, Amer. Meteor. Soc., Boston

Emanuel, K. A., 1986: An air-sea interaction theory for tropical cyclones. Part I. J. Atmos. Sci. , 42, 1062-1071 , 1995: The behavior of a simple hurricane model using a convective scheme based on subcloud-layer entropy equilibrium. J. Atmos. Sci., 52, 3959-3968 , 1995: Sensitivity of tropical cyclones to surface exchange coefficients and a revised steady-state model incorporating eye dynamics. J. Atmos. Sci., 52, 39693976

, 1997: Some aspects of hurricane inner-core dynamics and energetics. J. Atmos. Sci., 54, 1014-1026 , 1999: Thermodynamic control of hurricane intensity. Nature, 401, 665-669 , 2000: A statistical analysis of tropical cyclone intensity. Mon. Wea. Rev., 128, 1139-1152 
Gallacher, P. C., R. Rotunno and K. A. Emanuel, 1989: Tropical cyclogenesis in a coupled ocean-atmosphere model. Preprints of the 18th Conf. on Hurricanes and Trop. Meteor., Amer. Meteor. Soc., Boston

Hawkins, H. F. and S. M. Imbembo, 1976: The structure of a small, intense hurricaneInez 1966. Mon. Wea. Rev., 104, 418-442

Holland, G., 1980: Analytic model of the wind and pressure profiles in hurricanes. Mon. Wea. Rev., 108, 1212-1218

Khain, A. and I. Ginis, 1991: The mutual response of a moving tropical cyclone and the ocean. Beitr. Phys. Atmosph., 64, 125-141

Kleinschmidt, E., Jr., 1951: Gundlagen einer Theorie des tropischen Zyklonen. Archiv fur Meteorologie, Geophysik und Bioklimatologie, Serie A, 4, 53-72

Large, W. G. and S. Pond, 1982: Sensible and latent heat flux measurements over the ocean. J. Phys. Ocean., 12, 464-482

Lilly, D. K. and K. Emanuel, 1985: A steady-state hurricane model. Preprints of the 16th Conference on Hurricanes and Tropical Meteorology, Houston, TX, Amer. Meteor. Soc., Boston

Malkus, J. S., 1958: On the structure of the mature hurricane eye. J. Meteor. , 15, 337349.

Malkus, J. S. and H. Riehl, 1960: On the dynamics and energy transformations in steady-state hurricanes. Tellus, 12, 1-20

Miller, B. I., 1958: On the maximum intensity of hurricanes. J. Meteor., 15, 184-195

Ooyama, K., 1969: Numerical simulation of the life-cycle of tropical cyclones. J. Atmos. Sci., 26, 3-40

Powell, M. D., 1990: Boundary layer structure and dynamics in outer hurricane rainbands. Part I: Mesoscale rainfall and kinematic structure. Mon. Wea. Rev., 118, 891-917. , 1990: Boundary layer structure and dynamics in outer hurricane rainbands. Part II: Downdraft modification and mixed layer recovery. Mon. Wea. Rev., 118, 918938

Powell, M. D., P. J. Vickery and T. A. Reinhold, 2003: Reduced drag coefficients for high wind speeds in tropical cyclones. Nature, 422, 279-283

Raymond, D. J., 1995: Regulation of moist convection over the west Pacific warm pool. J. Atmos. Sci., 52, 3945-3959

Riehl, H., 1950: A model for hurricane formation. J. Appl. Phys., 21, 917-925 
, 1954: Tropical Meteorology. McGraw-Hill, New York, 392 pp.

1963: Some relations between wind and thermal structure of steady state hurricanes. J. Atmos. Sci., 20, 276-287

Riehl, H. and J. S. Malkus, 1961: Some aspects of Hurricane Daisy, 1958. Tellus, 13, 181-213

Rosenthal, S. L., 1971: The response of a tropical cyclone model to variations in boundary layer parameters, initial conditions, lateral boundary conditions and domain size. Mon. Wea. Rev., 99, 767-777.

, 1978: Numerical simulation of tropical cyclone development with latent heat release by resolvable scales. I: Model description and preliminary results. $J$.

Atmos. Sci., 35, 258-271

Rotunno, R. and K. A. Emanuel, 1987: An air-sea interaction theory for tropical cyclones. Part II. J. Atmos. Sci., 44, 542-561

Schade, L. R., 1997: A physical interpretation of SST-feedback. Preprints of the 22nd Conf. Hurricanes and Tropical Meteor., Boston, Amer. Meteor. Soc.

Schade, L. R. and K. A. Emanuel, 1999: The ocean's effect on the intensity of tropical cyclones: Results from a simple coupled atmosphere- ocean model. J. Atmos. Sci., 56, 642-651

Shay, L. K., 1999: Upper ocean response to tropical cyclones. RSMAS Tech. Note 99003 Miami

Shutts, G. J., 1981: Hurricane structure and the zero potential vorticity approximation. Mon. Wea. Rev., 109, 324-329

Sutyrin, G. G. and A. P. Khain, 1984: Effect of the ocean-atmosphere interaction on the intensity of a moving tropical cyclone. Izvestiya, Atmospheric and Oceanic Physica, 20, 697-703 